\title{
Case Indexing Using PSO and ANN in Real Time Strategy Games
}

\author{
Peng Huo, Simon Chi-Keung Shiu, HaiBo Wang, and Ben Niu \\ Department of Computing, The Hong Kong Polytechnic University, Kowloon, \\ Hong Kong, China \\ csphuo@comp.polyu.edu.hk
}

\begin{abstract}
This paper proposes a case indexing method using particle swarm optimization (PSO) and artificial neural network (ANN) in a defense-style real time strategy (RTS) game. PSO is employed to optimize the placement of cannons to defend the enemy attack. The execution time of PSO ( $>100$ seconds) is unsatisfied for RTS game. The result of PSO is used as a case indexing of past experience to train ANN. After the training (approximately 30 seconds), ANN can obtain the best cannon placement within 0.05 second. Experimental results demonstrated that this case indexing method using PSO and ANN efficiently speeded up the whole process to satisfy the requirement in RTS game.
\end{abstract}

\section{Introduction}

In recent years, computer games have been developed rapidly and provide an ideal platform for artificial intelligence (AI) research. The application of AI improves the intelligence and attraction of games. Machine learning approach may be a better choice to introduce human-like behavior [1,2]. Previous studies used genetic algorithm (GA) in shooting game [3] and strategical game [4]. Louis [5] developed a case-injected GA in real time strategy (RTS) game.

The GA mimics the natural biological evolution and has been popular. However, previous studies have reported that the drawback of GA is its expensive computational cost, while one advantage of particle swarm optimization (PSO) is its algorithm simplicity [6, 7, 8].

PSO is similar to GA in a sense but employs different strategies and computational effort. PSO is a relatively recent heuristic search method whose mechanics are inspired by the swarming social behavior of species. PSO has been used to improve playing strategies in board games, such as game Seega [9], African Bao game [10], checker [11]. Our recent study compared the performance of PSO and GA in a tower defense game [12]. The result, however, indicated that the execution time of either PSO or GA is unsatisfied for RTS games.

Artificial neural network (ANN) represents the art of neural information processing. With the hybrid of PSO-ANN approach, we aim to design the games in which the computer players can acquire the knowledge automatically by learning from the previous cases iteratively and memorizing the successful experiences to handle future situations like human being behaves.

S. Chaudhury et al. (Eds.): PReMI 2009, LNCS 5909, pp. 106 115, 2009.

(C) Springer-Verlag Berlin Heidelberg 2009 


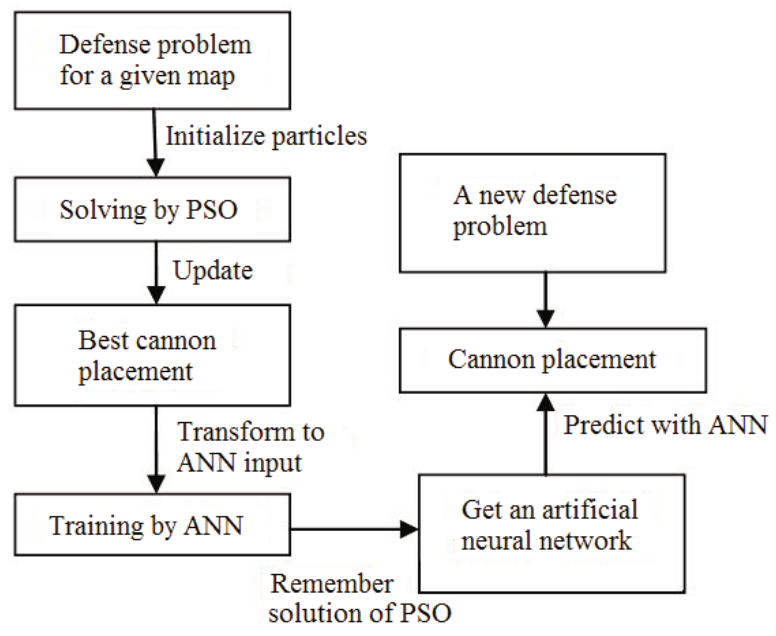

Fig. 1. Flowchart of game simulation

In this study, the optimum cannon placement in the battlefield obtained by PSO was used as a case indexing to train ANN. After training, ANN quickly obtained the optimum cannon placement in a new battlefield by consulting the case-base without going through the PSO process again. Figure 1 shows the flowchart of the game simulation.

\section{Methodology}

\subsection{Scenario of Tower Defense Game}

Similar to the concept of Forbus [13] spatial reasoning, battlefields can be divided into different small maps. In this study, the small maps are composed of defense base, barriers (mountains) and canyons. Canyon is the valley between hills or mountains and can be travelled through by the enemy. The number of canyons could be one, two, three, and four or determined randomly in the small maps as shown in Figure 2A-E. Dividing the battlefield into small maps gives two advantages. First, the fitness function can give a better solution easily. Second, it can increase the efficiency of PSO. Open area without canyons was not consider as it will not affect the solution and it will increase the run time as the search space increases.

Each battlefield map is digitalized as a $50 \times 50$ matrix. The points in barriers are set with a value of -1 . In the areas of the barriers, the cannon cannot be placed. The points in the blank area (or the open area) are set with a value of 0 , in which the cannons can be placed. When one cannon is placed at a point, the value of this point will be altered to 1 .

A scenario of strategic defense game is chosen as a test bed (Figure 2F). The game description is as follows.

1. Two teams are created in the battlefield. One is the attack team (enemy). Another one is the defense team (player). 


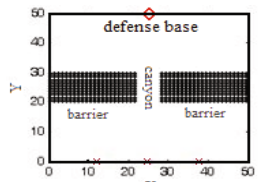

(A)

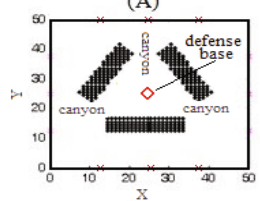

(C)

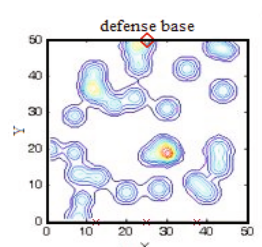

(E)

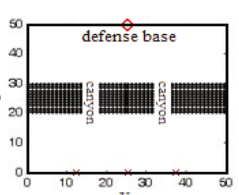

(B)

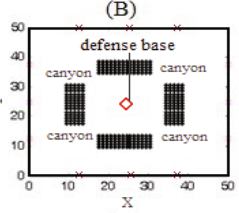

(D)

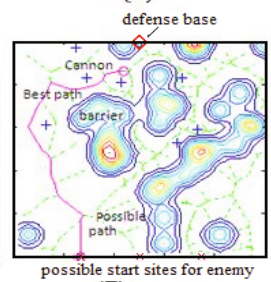

(F)

Fig. 2. Small battlefield maps with different canyons. (A) one canyon, (B) two canyons, (C) three canyons, (D) four canyons, (E) canyons determined randomly. (F) A base defense scenario. $\diamond$ : defense base, $\times$ : possible start sites of enemy, + : cannon, solid line: best path for enemy to travel, dashed line: possible path for enemy to travel.

2. The enemy must start from one of the possible start sites and move to the base of the defense team along one possible path with a minimum casualty (damage).

3. The defense team is able to place a number of cannons (e.g. seven cannons in this study) in the battlefield to kill the enemy when the enemy is approaching the defense base. Each cannon has a circle cannon-shot range.

4. The goal is to search an optimum cannon placement with a maximum casualty to the enemy no matter which possible path they choose.

\subsection{Learning Cases by Particle Swarm Optimization}

PSO is inspired by the social behavior of species such as a flock of migrating birds or a school of fish trying to reach an unknown destination. In PSO, a 'bird' in the flock is considered as a 'particle' presenting a candidate solution in the problem space. The particles in the swarm evolve their position and move to the destination based on their own experience and the experience of other particles [14]. Each particle has a memory to be capable of remembering the best position ever visited by it. Its best position with the best fitness is known as personal best, 'pbest' and the overall best position in the swarm is called as global best, 'gbest'.

The process starts from the initialization of the swarm with $\mathrm{N}$ random particles. Suppose that the search space is d-dimensional and the $i^{t h}$ particle is represented by a ddimensional vector $X_{i}=\left(x_{i 1}, x_{i 2}, \ldots, x_{i d}\right)$ and its velocity is denoted by $V_{i}=\left(v_{i 1}, v_{i 2}\right.$, $\left.\ldots, v_{i d}\right)$. And it can remember its best previously visited position $P_{i d}=\left(p_{i 1}, p_{i 2}, \ldots, p_{i d}\right)$. 
If the $g^{t h}$ particle is the best particle among all particles, its position is $P_{g d}$. Then, each particle updates its velocity to catch up with the best particle $g$ according to Eq. 1 and Eq. 2.

$$
\begin{gathered}
V_{i d}^{t+1}=\omega V_{i d}^{t}+c_{1} \gamma_{1}\left(P_{i d}^{t}-X_{i d}^{t}\right)+c_{2} \gamma_{2}\left(P_{g d}^{t}-X_{i d}^{t}\right) \\
X_{i d}^{t+1}=X_{i d}^{t}+V_{i d}^{t+1} \quad 1 \leq i \leq N
\end{gathered}
$$

where $\omega$ is inertia weight. $c_{1}$ and $c_{2}$ are cognitive acceleration and social acceleration, respectively, usually $c_{1}=c_{2}=2 . \gamma_{1}$ and $\gamma_{2}$ are random numbers uniformly distributed in the range $[0,1]$.

It is noted that the velocity update (Eq. 1) consists of three components, the previous velocity, the cognitive component, and the social component [15]. The first component is inertia component serving as a memory of the previous movement direction. The second component quantifies the current position with its own best position. The third component compares the current position with the best position in the swarm. Without the first part, all the particles will tend to move toward the same position, that is, the search area is contracting through the generations. Only when the global optimum is within the initial search space, there is a chance for PSO to find the solution. On the other hand, adding the first part, the particles will have a tendency to expand the search space, that is, they have the ability to explore the new area. So they more likely have global search ability. Both the local and global search will benefit solving problems. For different problems, there should be different balances between the local search ability and the global search ability. Considering of this, inertia weight $\omega$ is proposed to decrease linearly with generations from 1.4 to 0.5 determined by Eq. 3 [14].

$$
\omega_{i}=0.5+0.9(G-i) /(G-1) \quad i=1,2, \ldots, G
$$

where $G$ is the number of generations.

In this study, we used seven cannons as an example. The placement of the cannons is treated as a particle. Since the search space (battlefield) is 2-dimensional (X-Y coordinate), the position and velocity of the $i^{t h}$ particle are denoted by matrix $X_{i j d}$ and $V_{i j d}$, respectively, as follows.

$$
\begin{array}{cc}
X_{i j d}=\left\{x_{i j 1}, x_{i j 2}, \ldots, x_{i j d}\right\} & j=1,2 ; d=7 \\
V_{i j d}=\left\{v_{i j 1}, v_{i j 2}, \ldots, v_{i j d}\right\} & j=1,2 ; d=7
\end{array}
$$

where $j=1$ represents $\mathrm{x}$ direction and $j=2$ represents y direction.

To control the change of particles' velocities, upper and lower boundary is limited to a user-specified value of $V_{\max }$ [16], $V_{\max }=10$. In this study, the population size or the number of particles $(P)$ equals 50 and the number of generation or iteration cycles $(G)$ equals 100 .

In our simulation, a limit number of cannons are placed to give maximum damage to the enemy whatever the travelling path they choose. Base on the cannon placement, the best path to travel by the enemy is the one with minimum damage. The enemy will receive a damage value within the shot range of each cannon. The total damage $(D)$ is calculated by summing up all individual damages caused by different cannons (Eq. 4), 
and is used as the fitness value of this simulation. The higher the damage did to the enemy, the better the fitness of the cannon's positions. Cannon positions will be ranked by this fitness value to generate 'pbest' and 'gbest' particles.

$$
D=\sum_{k=1}^{N}\left(\frac{d_{i}}{v} \times p_{i}\right) \quad i=1,2, \ldots, n
$$

where $d_{i}$ is the distance that the enemy travels through the cannon-shot range of the $i^{t h}$ cannon. $v$ is the velocity of the enemy. $p_{i}$ is cannon power of the $i^{t h}$ cannon. $n$ is the number of cannons.

\subsection{Case Indexing for ANN}

In RTS games, one problem is that the long execution time of an optimization algorithm is unacceptable. To overcome this problem, we trained ANN using case indexing to speed up the whole process of optimization. Pal and Shiu [17] stated three principal advantages of using neural network to index cases compared with other traditional indexing methods, such as B+-tree, R-tree, and Bayesian Model. ANN first improves the case searching and retrieval efficiency. Secondly, it is very robust in handling noisy case data with incomplete or missing information which is the situation of strategy game. Finally, it is suitable for accumulating data in RTS games.

In our simulation, the PSO-obtained best solution of cannon placement in a given battlefield was used as a case input to ANN. It was supposed that every point of the best case had a circle range with a radius. The points in the circle were encoded as "-1" or "1". Then an encoding string was formed, where "-1" represents the barrier, "1" represents the open area and the final digit of the code,'d' represents the distance between the central point and the defense base. For example (Figure 4), eight points around Point A in the circle will be encoded as "-1" or "1". The 9-bit code string of Point A, [-1 $1-1-1-1-1-1-1$ d], is used as the input of ANN. It is noted that Point A will not be counted into the code string.

The network used in this study consisted of three layers, i.e. input layer, hidden layer, and output layer. The code string was the input of ANN. The number of the hidden layer was calculated as the square root of the length of the code string in back-propagation (BP) model neural network structure. In the above example, the number of the hidden layer is $\sqrt{9}=3$. The output of ANN was the probability of the central point to set up cannon. The higher value means the better location for setting up cannon.

The equation of the best location function (Eq. 5) is defined based on the relationship among the current landscape, the cannon locations obtained by PSO, and the distance to the defense base.

$$
f(A)=\sum_{k=1}^{N} e^{-\frac{\left(A_{1}-K_{k 1}\right)^{2}+\left(A_{2}-K_{k 2}\right)^{2}}{r}}
$$

where $f(A)$ is the best location function of Point A. $\left(A_{1}, A_{2}\right)$ is the coordinate of Point A. $N$ is the total number of cannons. $\left(K_{k 1}, K_{k 2}\right)$ is the coordinate of cannon $k$, and $r$ is a power decay coefficient for controlling the spread of $f(A)$ shown in Figure 3. 


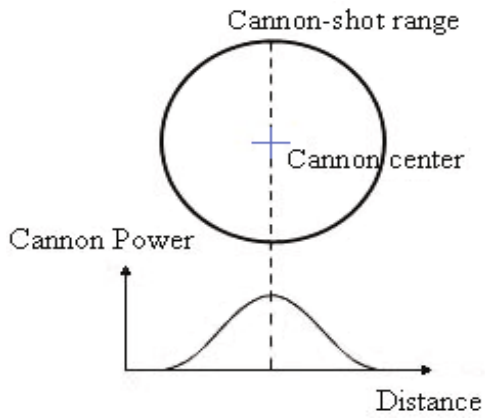

Fig. 3. Power decay coefficient

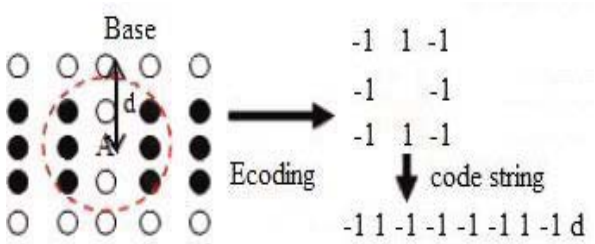

Fig. 4. Illustration of encoding of Point A for input to ANN

The code string and the best location value of each point in the PSO-obtained case were employed as input and output, respectively, to train the network. The ANN training was using BP and log-sigmoid output function. Then, the weight values of the hidden layer were settled. In a new battlefield map, the trained ANN quickly output the probability of each point to set up cannon when the surrounding terrain of the point was similar to the given battlefield in which the best case was obtained using PSO. Finally, the optimum cannon placement in the new battlefield map was obtained using ANN.

\section{Resutls}

Our simulations were performed using a computer with $2.1 \mathrm{GHz}$ CPU and 2 GB RAM under Windows XP. MathWorks Matlab 7.0 was used as the simulation tool.

\subsection{Fitness in Different Generations}

In our simulation, the evaluation function was based on the damage created on the enemy. Therefore, the fitness function is defined to be proportional to this damage value. Figure 5 shows the damage values as a function of generations measured in different maps. During the simulations, it was found that the damage value converged at approximately 50 generations. Figure 6 shows the final cannon placement in different maps after 100 generations. It is clearly seen that most of the cannons are placed in canyons, in which the cannons can make high damage to the enemy. 


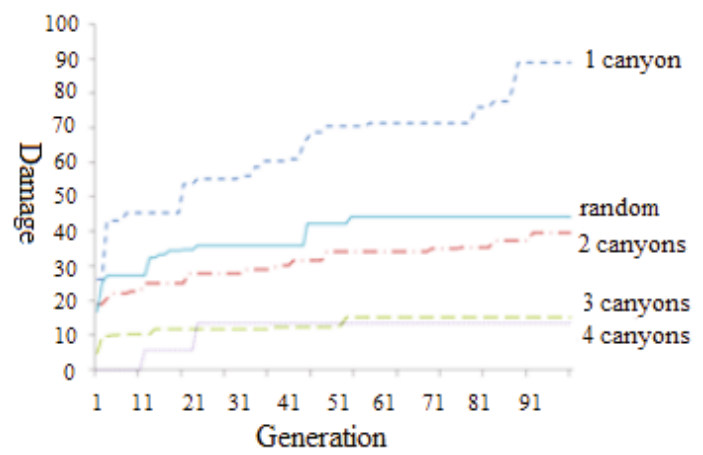

Fig. 5. Damage of the PSO-obtained cannons to the enemy as a function of generation in different maps
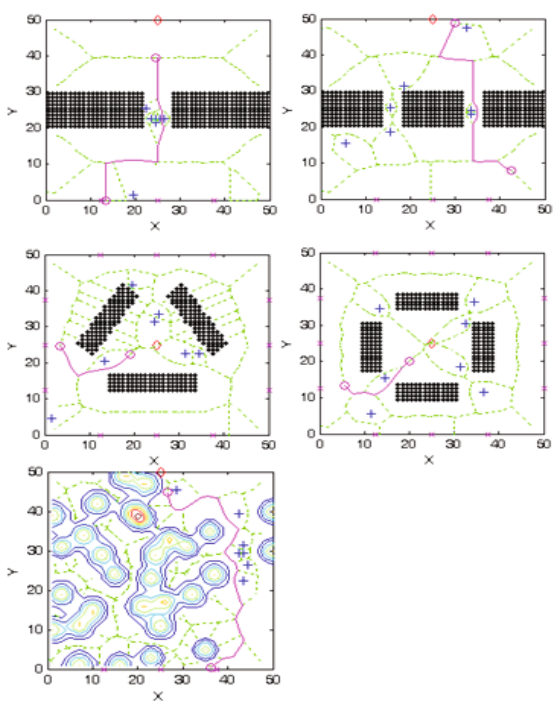

Fig. 6. PSO-obtained cannon placement in five battlefield maps

\subsection{Execution Time of PSO and Training and Execution Time of ANN}

Table 1 lists the execution time of PSO, the training time of ANN and the execution time of ANN for different maps. The maximum execution time of PSO is up to 360 seconds, which is obviously unacceptable for RTS games. With the application of case indexing for ANN, neural network could learn from the past experience (the PSO optimization result as case indexing) to speed up the searching process. After ANN training (approximately 30 seconds), our machine learning component became very useful. ANN could place the cannons in a new battlefield within less than 0.05 seconds that meets the requirement of RTS games. Figure 7A shows the points with the higher probability 
Table 1. Execution time of PSO, training time of ANN, and execution time of ANN (Cannon number: 7, Population size: 50, Generation number: 100; ANN model: BP)

\begin{tabular}{|c|c|c|c|}
\hline Map & $\begin{array}{c}\text { Execution } \\
\text { time } \\
\text { of } \mathrm{PSO}(\mathrm{s})\end{array}$ & $\begin{array}{c}\text { Training } \\
\text { time } \\
\text { of ANN (s) }\end{array}$ & $\begin{array}{c}\text { Execution } \\
\text { time } \\
\text { of ANN(s) }\end{array}$ \\
\hline 1 canyon & 126.3 & 26.0 & 0.03 \\
\hline 2 canyons & 132.6 & 26.2 & 0.03 \\
\hline 3 canyons & 322.1 & 30.3 & 0.04 \\
\hline 4 canyons & 309.0 & 28.7 & 0.04 \\
\hline random & 360.0 & 18.3 & 0.02 \\
\hline
\end{tabular}
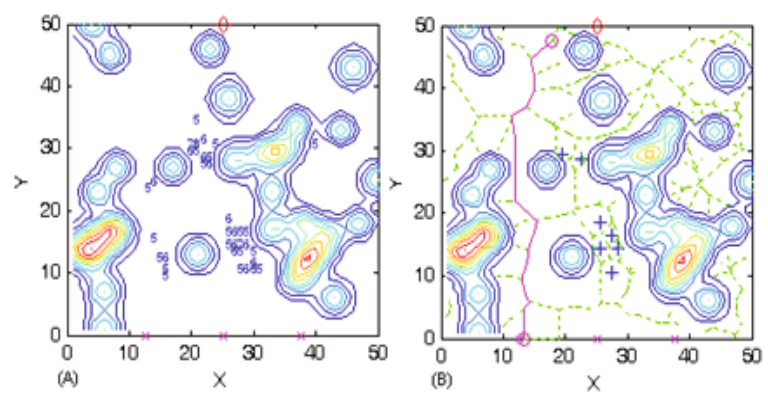

Fig. 7. (A) The points with higher probability $(\geq 0.5)$ predicted by ANN. The value of probability displayed in the map equals to probability $\times 10$. (B) Final cannon placement obtained by ANN and the best path travelled by enemy with minimum damage.

Table 2. Training time and recalling time of BP and RB ANN models (Cannon number: 7, Population size: 50, Generation number: 100)

\begin{tabular}{|c|c|c|}
\hline $\begin{array}{c}\text { Neural } \\
\text { network } \\
\text { system }\end{array}$ & $\begin{array}{c}\text { Training } \\
\text { time of } \\
\text { ANN (s) }\end{array}$ & $\begin{array}{c}\text { Recalling } \\
\text { time of } \\
\text { ANN (s) }\end{array}$ \\
\hline BP & 21.5 & 0.02 \\
\hline RBF(NEWGRNN) & 17.8 & 0.02 \\
\hline RBF(NEWRB) & 10.9 & 0.04 \\
\hline RBF(NEWRBE) & 12.4 & 10.2 \\
\hline
\end{tabular}

of setting up cannons predicted by ANN. Figure 7B shows the final cannon placement and the best path that the enemy travels with minimum damage.

\subsection{Training and Recalling Time of BP and RB networkN}

BP and Radial Basis (RB) network models have been commonly used in neural network systems. In our MATLAB simulations, three RB functions (RBF), NEWGRNN, NEWRB and NEWRBE, were used to design RB network. Figure 8 shows similar 

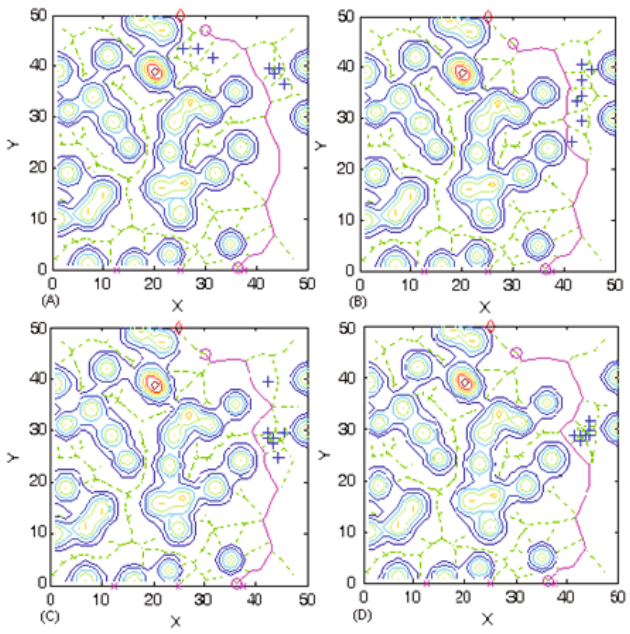

Fig. 8. Cannon placement obtained by ANN. (A) BP network; (B-D) RB networks using NEWGRNN (B), NEWRB (C) and NEWRBE (D) function.

results of cannon placement using BP and RBF. The main difference is in the training time and recalling time. In Table 2, RB network models show an improvement in the training time of ANN with a range from $17 \%$ to $49 \%$. Previous study of Wang and He [18] reported a 10\% improvement in Text Classification. It is shown that RB networks have a better improvement in comparison with BP. However, the recalling time of RB networks is averagely much more than that of BP. The difference in the recalling time may be a heavy workload as RTS games need to complete each game cycle in every 0.02 to 0.03 seconds. Therefore, we suggest using BP for RTS games due to its faster recalling time.

\section{Conclusion}

In this study, PSO is employed to optimize the placement of cannons to defend the enemy attack. The execution time of PSO ( $>100$ seconds) is unsatisfied for RTS game. We proposed a hybrid PSO-ANN method using the result of PSO as a case indexing of past experience to train ANN. After the training (approximately 30 seconds), ANN can quickly obtain the cannon placement within 0.05 seconds. Experimental results demonstrated that this case indexing method using PSO and ANN efficiently speeded up the whole process to satisfy the requirement in RTS game. Compared with RB network models, BP model with a faster recalling time and similar performance is suggested in RTS games.

\section{Acknowledgements}

This work is supported by the Hong Kong Polytechnic University A-PA6N and RGLS $\mathrm{PhD}$ research grants. 


\section{References}

1. Hsieh, J.L., Sun, C.T.: Building a player strategy model by analyzing replays of real-time strategy games. In: Neural Networks, IJCNN (2008)

2. David, M., Bourg, G.S.: AI for Game Developers. O'Reilly Media, Inc., Sebastopol (2004)

3. Cole, N., Louis, S.J.: Using a genetic algorithm to tune first-person shooter bots. In: Proceedings of IEEE Congress on Evolutionary Computation, Portland, OR, pp. 139-145 (2004)

4. Salge, C., Lipski, C.: Using genetically optimized artificial intelligence to improve gameplaying fun for strategical games. In: Proceedings of the 2008 ACM SIGGRAPH symposium on Video Games. ACM Press, New York (2008)

5. Louis, S.J., Miles, C.: Playing to learn: case-injected genetic algorithms for learning to play computer games. IEEE Transactions on Evolutionary Computation 9(4), 669-681 (2005)

6. Elbeltagi, E., Hegazy, T., Grierson, D.: Comparison among five evolutionary-based optimization algorithms. Advanced Engineering Informatics 19(1), 43-53 (2005)

7. Edwards, A., Engelbrecht, A.P.: Comparing Particle Swarm Optimisation and Genetic Algorithms for Nonlinear Mapping. In: Proceedings of IEEE Congress on Evolutionary Computation, Vancouver, BC, Canada, pp. 694-701 (2006)

8. Ou, C., Lin, W.X.: Comparison between PSO and GA for Parameters Optimization of PID Controller. In: Proceedings of the 2006 IEEE International Conference on Mechatronics and Automation, Luoyang, China, pp. 2471-2475 (2006)

9. Abdelbar, A.M., Ragab, S., Mitri, S.: Applying Co-Evolutionary Particle Swam Optimization to the Egyptian Board Game Seega. In: Proceedings of the First Asian-Pacific Workshop on Genetic Programming, Canberra, Australia, pp. 9-15 (2003)

10. Conradie, J., Engelbrecht, A.P.: Training Bao Game-Playing Agents using Coevolutionary Particle Swarm Optimization. In: IEEE Symposium on Computational Intelligence and Games, Reno/Lake Tahoe, USA, pp. 67-74 (2006)

11. Franken, N., Engelbrecht, A.: Comparing PSO structures to learn the game of checkers from zero knowledge. In: Proceedings of IEEE Congress on Evolutionary Computation, Canberra, Australia, pp. 234-241 (2003)

12. Huo, P., Shiu, S.C.K., Wang, H.B., Niu, B.: Application and Comparison of Particle Swarm Optimization and Genetic Algorithm in Strategy Defense Game. In: The 5th International Conference on Natural Computation, Tianjin, China, August 2009, pp. 14-16 (2009)

13. Forbus, K.D., Mahoney, J.V., Dill, K.: How qualitative spatial reasoning can improve strategy game AIs. IEEE Intelligent Systems 17(4), 25-30 (2002)

14. Shi, Y., Eberhart, R.: A modified particle swarm optimizer. In: Proceedings of IEEE Conference on Evolutionary Computation, Anchorage, AK, pp. 69-73 (1998)

15. Engelbrecht, A.P.: Fundamentals of Computational Swarm Intelligence. John Wiley-Sons, Ltd, Chichester (2005)

16. Kennedy, J., Eberhart, R.: Particle swarm optimization. In: Proceedings of IEEE International Conference on Neural Networks, Perth, Australia (1948)

17. Pal, S.K., Shiu, S.C.K.: Foundations of Soft Case-Based Reasoning. Wiley Interscience, Hoboken (2004)

18. Wang, Z., He, Y.: A Comparison among Three Neural Networks for Text Classification. In: Proceedings of 8th International Conference on Signal Processing (2006) 